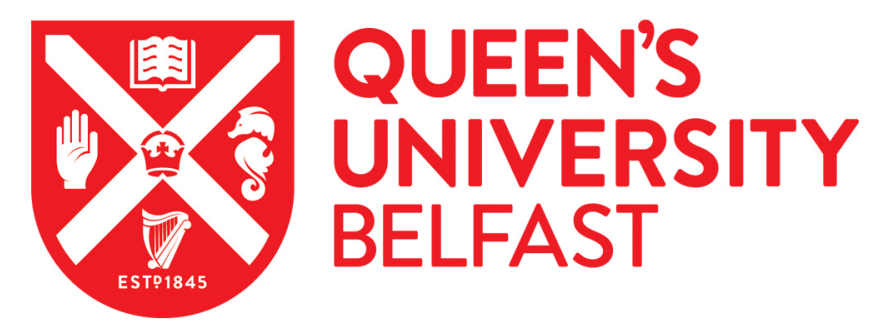

\title{
Phosphorylation Mechanism of Phosphomevalonate Kinase: Implications for Rational Engineering of Isoprenoid Biosynthetic Pathway Enzymes
}

Huang, M., Wei, K., Li, X., McClory, J., Hu, G., Zou, J-W., \& Timson, D. J. (2016). Phosphorylation Mechanism of Phosphomevalonate Kinase: Implications for Rational Engineering of Isoprenoid Biosynthetic Pathway Enzymes. Journal of Physical Chemistry B, 120(41), 10714-10722. https://doi.org/10.1021/acs.jpcb.6b08480

Published in:

Journal of Physical Chemistry B

Document Version:

Peer reviewed version

Queen's University Belfast - Research Portal:

Link to publication record in Queen's University Belfast Research Portal

\author{
Publisher rights \\ (C) 2016 American Chemical Society \\ This document is the Accepted Manuscript version of a Published Work that appeared in final form in \\ Journal of Physical Chemistry B, copyright (C) American Chemical Society after peer review and technical editing by the publisher. \\ To access the final edited and published work see http://pubs.acs.org/doi/abs/10.1021/acs.jpcb.6b08480
}

\section{General rights}

Copyright for the publications made accessible via the Queen's University Belfast Research Portal is retained by the author(s) and / or other copyright owners and it is a condition of accessing these publications that users recognise and abide by the legal requirements associated with these rights.

Take down policy

The Research Portal is Queen's institutional repository that provides access to Queen's research output. Every effort has been made to ensure that content in the Research Portal does not infringe any person's rights, or applicable UK laws. If you discover content in the Research Portal that you believe breaches copyright or violates any law, please contact openaccess@qub.ac.uk. 


\title{
Phosphorylation Mechanism of Phosphomevalonate Kinase: Implications for Rational Engineering of Isoprenoid Biosynthetic Pathway Enzymes
}

\author{
Meilan Huang, ${ }^{*}{ }^{\dagger}$ Kexin Wei, ${ }^{\dagger}$ Xiao Li, ${ }^{\dagger}$ James McClory, ${ }^{\dagger}$ Guixiang Hu, ${ }^{\ddagger}$ Jian-Wei Zou, ${ }^{\ddagger}$ \\ David Timson $\xi$
}

${ }^{\dagger}$ School of Chemistry and Chemical Engineering, Queen's University Belfast, David Keir Building, Stranmillis Road, Belfast, BT9 5AG, Northern Ireland, United Kingdom ${ }^{\ddagger}$ School of Biotechnology and Chemical Engineering, Ningbo Institute of Technology, Zhejiang University, Ningbo 315100, China

${ }^{\xi}$ School of Pharmacy and Biomolecular Sciences, The University of Brighton, Huxley Building. Lewes Road, Brighton, BN2 4GJ, United Kingdom

Corresponding author

*Meilan Huang

Phone: +442890974698.

Fax: +44 2890976524 .

E-mail: m.huang@qub.ac.uk. 


\begin{abstract}
Mevalonate pathway is of important clinical, pharmaceutical and biotechnological relevance. However, lack of the understanding of the phosphorylation mechanism of the kinases in this pathway has limited rationally engineering the kinases in industry. Here the phosphorylation reaction mechanism of a representative kinase in the mevalonate pathway, phosphomevalonate kinase, was studied by using molecular dynamics and hybrid QM/MM methods. We find that a conserved residue (Ser106) is reorientated to anchor ATP via a stable H-bond interaction. In addition, Ser213 located on the $\alpha$-helix at the catalytic site is repositioned to further approach the substrate, facilitating the proton transfer during the phosphorylation. Furthermore, we elucidate that Lys101 functions to neutralize the negative charge developed at the $\beta$-, $\gamma$-bridging oxygen atom of ATP during phosphoryl transfer. We demonstrate that the dissociative catalytic reaction occurs via a direct phosphorylation pathway. This is the first study on the phosphorylation mechanism of a mevalonate pathway kinase. The elucidation of the catalytic mechanism not only sheds light on the common catalytic mechanism of GHMP kinase superfamily, but also provides the structural basis for engineering the mevalonate pathway kinases to further exploit their applications in the production of a wide range of fine chemicals such as biofuels or pharmaceuticals.
\end{abstract}




\section{Introduction}

The GHMP kinase superfamily is named after Galactokinase (GALK), Homoserine kinase (HK), Mevalonate kinase (MK) and Phosphomevalonate kinase (PMK). ${ }^{1}$ The superfamily, now including over 800 protein sequences, is defined by three conserved sequence motifs (IIII). Despite the conserved sequence, the structures of GHMP kinases show some differences with diverse $\mathrm{Mg}$ coordination environments and a range of substrates acting as the $\gamma$ phosphate acceptor during the phosphorylation reaction. Based on the structural features of the active sites of GHMP kinases, two catalytic mechanisms have been proposed. The first mechanism is a common catalytic mechanism where a negatively charged residue (aspartate) acts as a catalytic base, assisting proton abstraction from the hydroxyl group of the substrate. As a result, a more strongly nucleophilic alkoxide ion is formed, which in turn attacks the $\gamma$ phosphate of ATP. Meanwhile, a positively charged residue such as lysine or arginine is often observed in the active site. These are postulated to stabilise the negative charges developed during phosphorylation. ${ }^{2}$ The catalytic base mechanism was suggested for MK, GALK and diphosphomevalonate decarboxylase (DPM-DC) based on site-directed mutagenesis studies. 3-7 The second mechanism was proposed for homoserine kinase (HK), where asparagine and threonine residues replace the aspartate and lysine respectively. Since there is no potential catalytic base present, the hydroxyl group of the substrate was proposed to directly attack the $\gamma$-phosphate of ATP assisted by enzyme-mediated stabilisation of the transition state. ${ }^{8,9}$ Recently, a combined QM/MM calculation was used to investigate the reaction mechanism of human GALK. ${ }^{10}$ It was found that the reaction occurs via direct a phosphorylation mechanism instead of a base catalyzed mechanism. However, whether the direct phosphorylation mechanism is common for all GHMP kinases is still yet to be determined. GHMP kinase family participates in several essential metabolic pathways, among which the mevalonate (MVA) pathway is of important clinical, pharmaceutical and biotechnological relevance. ${ }^{11}$ The MVA pathway is composed of three GHMP kinases, MK, PMK and diphosphomevalonate decarboxylase (DPM-DC). ${ }^{12}$ The product of the MVA pathway, isopentenyl diphosphate (IPP), is the building block of isoprenoids, the largest class of natural products and comprising an extremely diverse range of chemical structures. Due to the ubiquity and metabolic significance of isoprenoids, it is not surprising that excess or deficiency in the isoprenoid synthesis can cause cellular dysfunction and various diseases. e.g. The MVA pathway is essential for the survival of Streptococcus pneumonia in the lung and serum. ${ }^{13}$ It was reported that the product of PMK, diphosphomevalonate (DPM), feed- 
back inhibits MK in S. pneumonia. Therefore, regulation of the MVA pathway would provide novel therapies to intervene in the pathway to inhibit the devastating effect of the organism. Recently, inhibitors of GHMP kinases in the mevalonate pathway of S. pneumoniae were reported. ${ }^{14-16}$

Isoprenoids have been recognized as anticancer and antimalarial drugs ${ }^{17,18}$ as well as potential gasoline replacements. ${ }^{19,20}$ Chemical synthesis or modification of isoprenoids is difficult due to their structural complexity, and therefore the large scale of production of isoprenoids in industry is hindered. Metabolic engineering of the MVA pathway provides an alternative approach to traditional synthesis of these natural products. ${ }^{21}$ A number of metabolic engineering studies have been reported to optimize the enzymes in this pathway to improve the yields of isoprenoids in Escherichia coli. ${ }^{22-27}$

Elucidation of the catalytic mechanism of the kinases involved in MVA pathway can help to reveal the common features in the catalytic mechanisms shared by GHMP superfamily kinases, which would shed light on the development of antibiotic therapies that target the bacterial enzymes involved in the MVA pathway. It may also provide guidance for rationally engineering the kinases for industrial applications such as the more efficient production of isoprenoid-based biofuels. PMK, a representative kinase in the MVA pathway, is a special GHMP kinase that has a negatively charged phosphate as the acceptor of the phosphorylation. Different from most GHMP kinases where $\mathrm{Mg}^{2+}$ is coordinated to more than one phosphoryal groups of ATP, the magnesium ion in PMK is coordinated to both the $\gamma$-phosphate of ATP and the phosphoryal group of the substrate. In the present research, the reaction mechanism of PMK was investigated by molecular dynamics (MD) simulations and hybrid QM/MM calculations.

\section{Methods}

\subsection{Protein Preparation}

The crystal structure of PMK from S. pneumoniae in complex with its substrate $(R)-5-$ phosphomevalonate (PMV) and AMPPNP· $\mathrm{Mg}^{2+}$ (PDB code: 3GON) was used as the initial structure. ${ }^{28}$ The nitrogen atom bridging the $\beta$ - and $\gamma$ - phosphate groups in AMPPNP was substituted with an oxygen atom, yielding an ATP molecule. Three structural water molecules coordinated to the magnesium ion were kept in the structure. 


\subsection{Molecular Dynamics Simulations}

The parameters of PMV were prepared by single point charge calculation using Gaussian 09 29 at the level of HF/6-31G (d). Then RESP charges were generated using antechamber 30 encoded in Amber 10. ${ }^{31}$ The partial atomic charges of PMV and ATP were calculated separately, while atom type and charge of magnesium ion was directly utilized from Amber Parm99 force field. ${ }^{32}$

A TIP3P water box was added in the protein system with the minimum distance between the box wall and protein of $8 \AA \AA$. A total of 8,563 water molecules were added. In addition, 13 sodium ions were added to maintain the neutrality of the system. The solvate including the protein, ATP and the substrate PMV were restrained with a force constant $0.5 \mathrm{kcal} \cdot \mathrm{mol}^{-1} \cdot \AA^{-2}$, while water molecules were set free in order to remove abnormal van der Waals forces between the protein and surrounding water molecules. In total 5,000 steps of constrained minimization were performed comprised of 2,500 steps steepest descent minimization followed by 2,500 steps conjugate gradient minimization.

MD simulations were performed for the wild-type (WT) kinase and five variants, K9R, K9M, K101R, K101M and A293T respectively. After minimisation, the protein system was heated slowly from $0 \mathrm{~K}$ to $300 \mathrm{~K}$ with a time step of $1 \mathrm{fs}$ over $500 \mathrm{ps}$. A periodic boundary condition was applied, and equilibration was carried out with NVT ensemble at $300 \mathrm{~K}$ for another 500 ps. The Particle Mesh Ewald method was used to calculate the long-range electrostatic interactions with the non-bonded van der Waals force being set to $10 \AA .{ }^{33}$ The SHAKE method was used to constrain covalent bonds involving hydrogen atoms. ${ }^{34} \mathrm{~A}$ molecular dynamics (MD) simulation (10 ns) was run for the wild-type (WT) enzyme and each of the variant systems with a time step of 1 fs using NPT ensemble under a reference pressure of 1 atm and temperature of $300 \mathrm{~K}$. Clustering analysis was performed for the last nanosecond trajectory of MD simulation to obtain the representative conformation of the kinase. ${ }^{35}$

\subsection{QM/MM calculations}

The protein complexes were optimized using the two-layer QM/MM method implemented in ONIOM. The energy is calculated by Eq. 1:

$$
E_{\text {real }}^{\text {ONIOM }}=E_{\text {model }}^{\text {high }}+E_{\text {real }}^{\text {low }}-E_{\text {model }}^{\text {medium }}
$$


The high layer was treated with a quantum mechanics (QM) method and the low layer was treated with a molecular mechanics (MM) method. The entire molecule was denoted as the real system. The boundary between QM and MM region was treated using hydrogen atoms as link atoms. The density functional theory with the B3LYP-D method and 6-31G(d) basis set was used to calculate the QM region. The rest of the system was treated using AMBER Parm99 force field. ${ }^{32}$

The electrostatic interaction between the QM and MM regions were optimized by using electronic embedding, which incorporates the partial charges of the MM region into the quantum mechanical Hamiltonian and allows the QM wavefunction to be polarised. ${ }^{36}$ MerzKollman-Singh approximate charge was used during optimization microiterations with electronic embedding. ${ }^{37,38}$

The QM region included ATP, PMV, Ala293, Asp297, Lys9, Lys101, Ser213 and three water molecules that were coordinated to the Mg ion (Figure 1). ATP was truncated to keep the triphosphate arm. There were 83 atoms in total in the QM region and the total charge of the QM system was -3. Only the side chains of the residues in the QM region were retained to compromise between the time-consuming nature of the electronic embedding scheme and the accuracy of the calculation. ${ }^{39}$ A reduced system within $12 \AA$ of the active site was subjected to a preliminary minimization, while the residues within $6 \AA$ of the active site were allowed to move and the rest of the system was kept frozen.

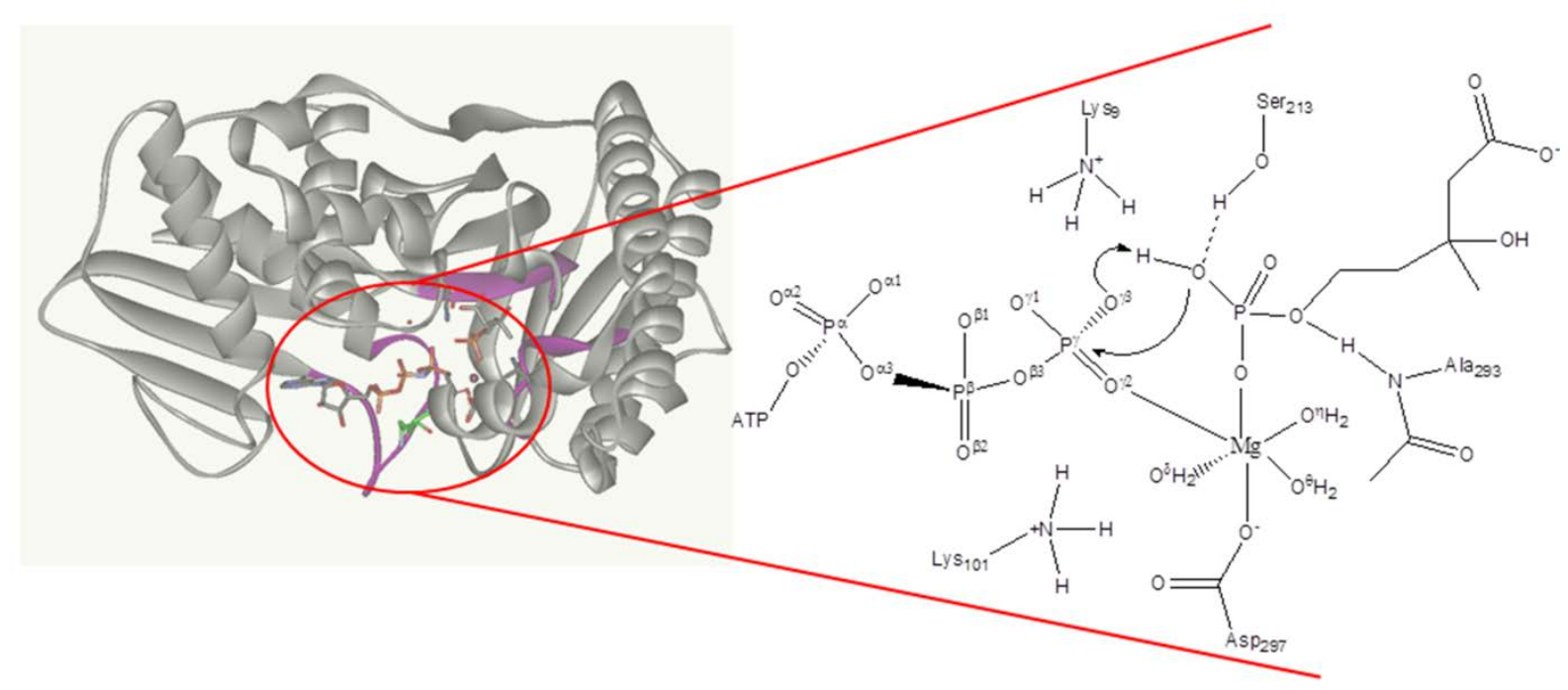

Figure 1. The QM region (highlighted in red cycle) of $\mathrm{PMK}$ in the $\mathrm{QM} / \mathrm{MM}$ calculation

\section{Results and Discussion}




\subsection{Identification of key residues in the catalytic reaction pathway}

PMK catalyzes the second step of the MVA pathway. It transfers the $\gamma$-phosphoryl group of ATP to the phosphoryl group of PMV, yielding (R)-5-diphosphomevalonate (DPM) (Figure 2). Similar to other GHMP superfamily kinases, PMK contains three conserved sequence motifs (I-III) ${ }^{1}$ (Figure 3). The most conserved motif II has a sequence of Pro-X-X-Gly-Leu$\mathrm{X}$-Ser-Ser-Ala, which is folded into the phosphate binding loop or p-loop.<smiles>CC(O)(CCO)CC(=O)O</smiles>

mevalonate<smiles>CC(O)(CCOP(=O)([O-])[O-])CC(=O)OI</smiles>

mevalonate-5-phosphate

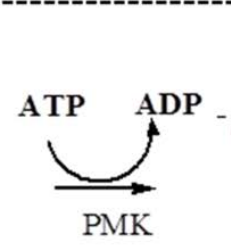

PMK

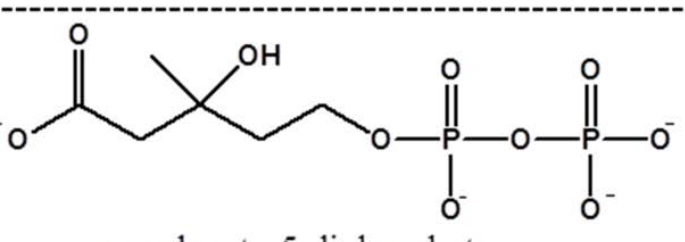

mevalonate-5-diphosphate

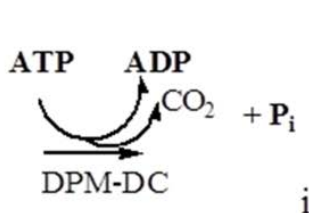<smiles>C=C(C)CCOP(=O)([O-])OP(=O)([O-])[O-]</smiles>

isopentenyl diphosphate

(IPP)

Figure 2. The mevalonate pathway: The phosphorylation of mevalonate-5-phosphate (highlighted in dashed box) is catalysed by PMK. 
The crystal structure of PMK from S. pneumoniae showed that a $\mathrm{Mg}^{2+}$ ion is coordinated to the $\gamma$-phosphoryl group of ATP and the phosphoryl group of the substrate, as well as to Asp297 and three structural water molecules (Figure 4A). A highly conserved residue, Lys9 located in the motif I, forms strong ionic interactions with the PMV phosphoryl group and the $\gamma$-phosphoryl group of ATP, stabilizing the substrate and nucleotide along the reaction path. This is in line with previous studies on the effect of the mutation of Lys9 on the affinity for PMV. ${ }^{40}$ Lys101, located at the end of the p-loop, is in close proximity to the $\beta$-, $\gamma$ - bridging oxygen. Comparison of the ligand-bound and apo PMK structures disclosed the repositioning of this residue to stabilize the negative charge developed during phosphoryl transfer. ${ }^{28}$ Ser107, also located in the motif II p-loop, is H-bonded to the $\beta$-phosphate of ATP, although there have been no direct mutagenesis studies that address its role in substrate recognition or turnover. In addition, the backbone of Ala293 is H-bonded to the phosphoryl group of PMV. Mutation of Ala293 selectively increased the Michaelis constant for PMV (KM,PMV) 17,000fold and decreased the turnover number $\left(k_{\text {cat }}\right)$ considerably (64 fold), indicating that it plays a significant role in catalysis by stabilizing the phosphate group of PMK during the reaction. ${ }^{40}$ Ser213, located on an $\alpha$-helix at the catalytic site, forms a H-bond with one of the coordinated water molecules, as well as the phosphoryl group of PMV, although mutation of this residue only modestly decreased the $k_{\text {cat. }}{ }^{40}$

The catalytically important residues may execute their functions via communication with distant residues. ${ }^{41} \mathrm{MD}$ simulation enables the entire protein structure including the distant residues as well as the active site residues to evolve with time in response to the mutations. In order to elucidate the role of these residues during the catalytic reaction and locate the starting structure of the reactant to be used in the subsequent diabatic potential energy surface scan, a 10-ns MD simulation was performed on the PMK in complex with ATP, the substrate and $\mathrm{Mg}^{2+}$ ion.

Distinct from other GHMP kinases, PMK catalyzes the transfer of the $\gamma$-phosphate of ATP to another negatively charged phosphate in the substrate. In addition, $\mathrm{Mg}^{2+}$ is only coordinated to the $\gamma$-phosphoryl group of ATP in PMK, whereas it is coordinated to at least two phosphoryl groups of ATP in other GHMP kinases. ${ }^{10}$ Prior to the MD simulations and potential energy surface scan, we searched and examined the structures of diverse kinases for which the phosphorylation mechanisms have been elucidated to decide the protonation state of the substrate. Human galactokinase, a member of GHMP family, follows a direct phosphorylation mechanism, where Arg228 was found to move close to, and stabilize, the 
negative charge developed at the $\beta$-, $\gamma$ - bridging oxygen atom of the ATP during bond cleavage (supporting information Figure S1). ${ }^{10}$ The proton of C1 hydroxyl group of galactose transfers to the $\gamma$-phosphate oxygen atom of ATP and the phosphoryl transfer coincides with the proton migration from galactose. ErbB3 kinase also follows a direct phosphorylation mechanism where the substrate (tyrosine) acts as a proton donor, donating a proton onto the $\gamma$-phosphate during phosphorylation. ${ }^{42}$ Ting Shi et al. disclosed a catalytic acid mechanism for CheA histidine kinase where the $\beta$-, $\gamma$ - bridging oxygen of ATP forms a hydrogen-bond network with two water molecules, which participate in the protein relay from the catalytic acid Lys48 to the $\beta$-, $\gamma$ - bridging oxygen of the ATP. ${ }^{43}$ A catalytic base mechanism was suggested for cyclin-dependent protein kinases, where Asp127 acts as a catalytic base to abstract a proton from the substrate serine, which attacks the $\gamma$-phosphate of ATP. ${ }^{44}$ Despite of the diversity of the reaction mechanisms of the kinases, proton transfer from the substrate hydroxyl group has been validated. Therefore, in our MD simulations, the oxygen $\left(\mathrm{O}^{\mathrm{P} 3}\right)$ of PMV was protonated such that the substrate has a hydroxyl group to donate a proton (Figure 3).

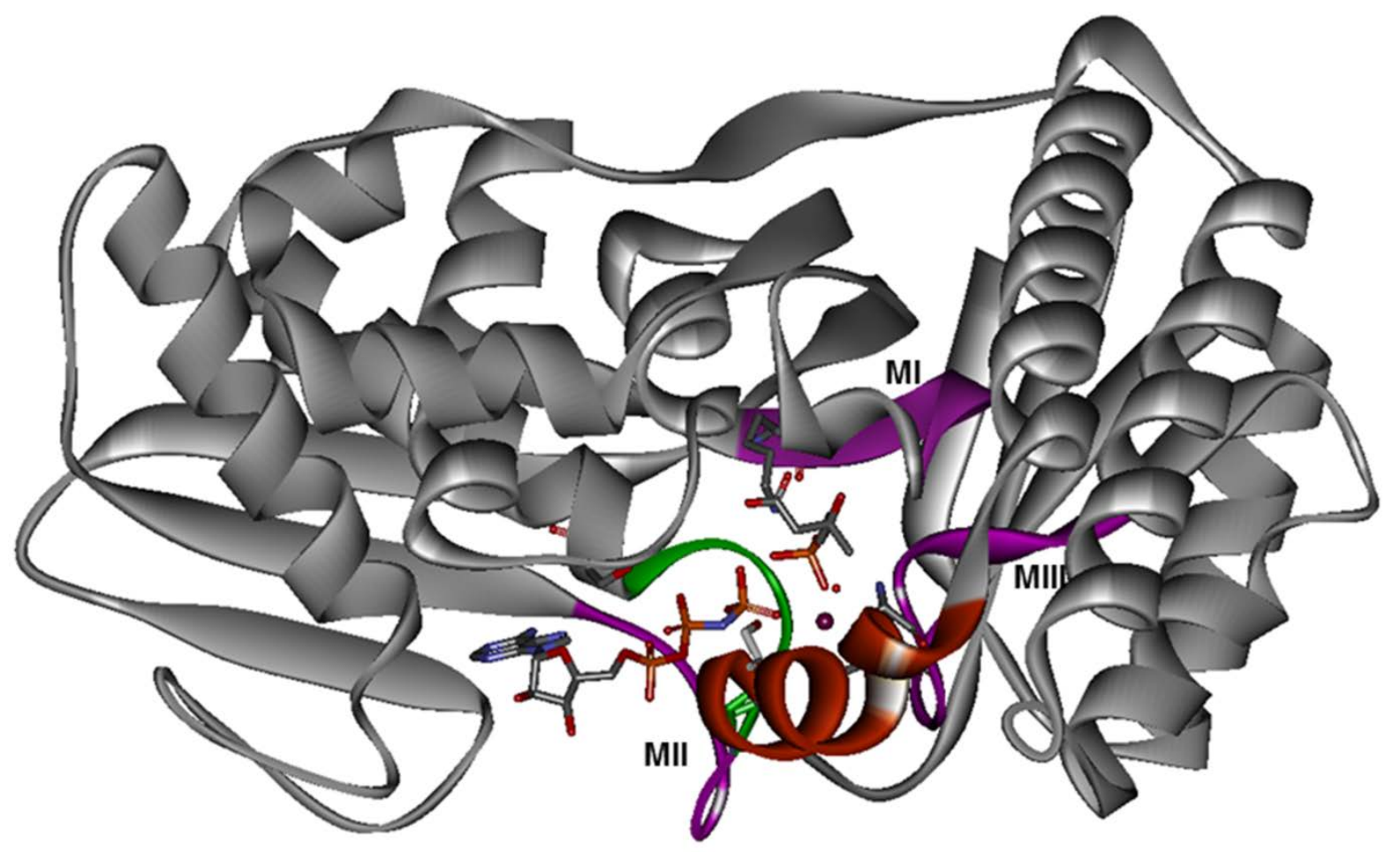


Figure 3. The highly conserved Motif II and p-loop in Streptococcus pneumoniae phosphomevalonate kinase (PDB ID: 3GON). ${ }^{28}$ Motifs I-III are shown in purple, p-loop is shown in green, and the $\alpha$-helix above the catalytic site is shown in red.

As time evolved, we found the configuration of the active site of the PMK complex observed in the crystal structure of PMK was well maintained throughout the MD simulation. The $\mathrm{Mg}^{2+}$ is octahedrally coordinated to Asp297, the phosphoryl group of PMV, the $\gamma$-phosphoryl group of ATP, as well as three structural water molecules. Lys9 is located between PMV and ATP, stabilizing the $\gamma$-phosphoryl group of ATP and the phosphoryl group of PMV, which in turn forms a H-bond with the backbone of Ala293. In the crystal structure of PMK, Ser213 located on the $\alpha$-helix at the catalytic site is positioned to form a $\mathrm{H}$-bond with a coordinating water, as well as the PMV phosphate. Interestingly, with time evolution in the MD simulation, the hydroxyl group of the Ser213 further approached the substrate phosphate, forming a stronger $\mathrm{H}$-bond with the substrate. As a result, the original $\mathrm{H}$-bond interaction with the structural water observed in the crystal structure was lost (Figure 4).

In a previous computational study on the mechanism of galactokinase, another GHMP kinase, the positively charged Arg228 functions to stabilize the negative charge developed at the $\beta, \gamma$ bridging oxygen of the ATP during bond cleavage. ${ }^{10}$ Two conserved catalytic residues (Ser141 and Ser142) in galactokinase stabilize ATP in the oxyanion hole. Although the coordination environment of the $\mathrm{Mg}^{2+}$ ion in the two GHMP kinases is different, we identified the corresponding functional residues in PMK by careful comparison of structures of galactokinase and PMK obtained from MD simulations. Analogous to Arg228 in galactokinase, Lys101 in PMK is H-bonded to the $\beta$-, $\gamma$ - bridging oxygen of ATP and could stabilize the negative charge which develops during phosphoryl transfer. When comparing the sequences of GHMP kinases, we found Lys101 in S. pneumoniae PMK is located on a unique KKF insertion at the beginning of the p-loop (Figure S2). In the crystal structure of PMK, Ser106 on motif II points away from the catalytic site while Ser107 is H-bonded to the $\beta$-phosphoryl group of ATP. Interestingly, with time evolution in the MD simulation, the hydroxyl group of Ser106 moves towards the oxygen atom of the $\alpha$-phosphoryl group of ATP to form a H-bond (Figure 4). Thus, Ser106, along with the adjacent serine Ser107, can be analogous to the two conserved serine residues on the p-loop of galactokinase, functioning to anchor the nucleotide in the catalytic oxyanion hole. 

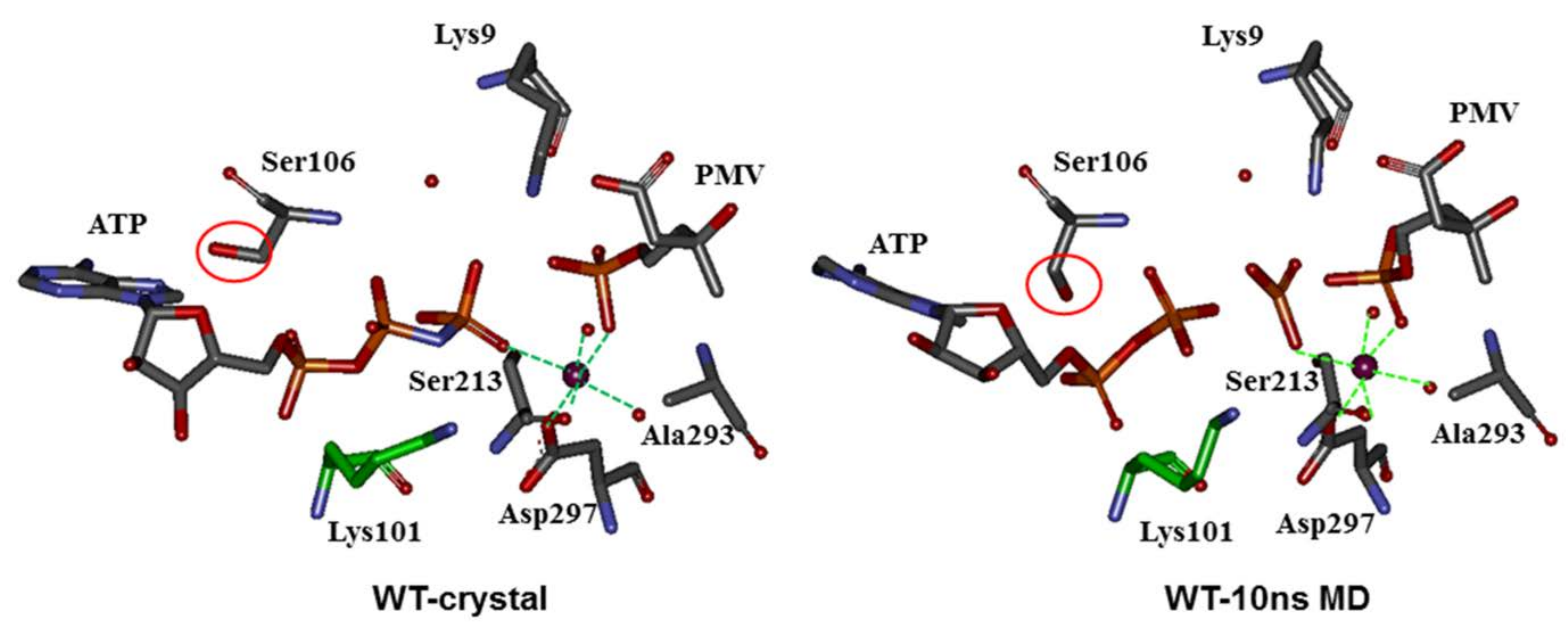

Figure 4. The catalytic centre of PMK. (A) The crystal structure of PMK: The $\mathrm{Mg}^{2+}$ ion is (in purple) in complex with ATP and PMV. (B) The simulated structure from 10 ns MD simulation.

\subsection{Effect of sequence alterations on $\mathrm{Mg}^{2+}$ coordination}

In order to understand the effect of alteration of key residues that may be involved in the reaction pathway of PMK, Lys9, Lys101 and Ala293 were altered and 10-15 ns MD simulations carried out. When the ammonium ion of the Lys 9 was replaced with the guanidinium group of arginine, a significant decrease of $k_{\text {cat }}$ was reported. ${ }^{40} \mathrm{~A}$ similar effect was observed while the alanine at the position 293 was substituted by threonine. Therefore, we simulated the corresponding variants K9R and A293T variants. Lys9 was also replaced by a neutral residue methionine, which has a similar length to lysine but couldn't establish electrostatic interactions with the phosphoryl groups of the substrate or ATP. The lysine at position101, which was suggested to stabilize the negative charge which develops during the breakage of the P-O bond between the $\beta$ and $\gamma$-phosphoryal groups of ATP, was also mutated into arginine and methionine, respectively.

In the MD simulation of K9R, the RMSD value of the protein backbone increased slightly as time elapsed and finally stabilized at $\sim 1.0$ Å from 4 ns onwards (Figure S3). This slight fluctuation indicates that there were no significant conformational changes in the protein over the time period studied. A representative structure of the last nanosecond (Figure 5) was compared to the wide-type complex structure. When the lysine residue at position 9 was changed to arginine, the coordination of $\mathrm{Mg}^{2+}$ was distorted. Two water molecules moved away resulting in an unstable conformation of the active site. 
In the MD simulation of K9M, the RMSD value of the protein backbone slightly increased from $0.8 \AA$ to $1.5 \AA$ in the first 3 ns and then stabilized to approximately $1.2 \AA$, indicating that the system remained stable and had reached equilibrium. The positively charged Lys9 is believed to stabilize phosphate group of both ATP and PMV during the reaction. When Lys9 was altered to the neutral amino acid methionine, one water molecule moved away from the active site yielding an unstable pentacoordinated $\mathrm{Mg}^{2+}$.

Previous studies on various kinases that contain an invariant lysine residue in their catalytic domains showed that this residue is actively involved in phosphorylation but is not required for anchoring ATP. ${ }^{45}$ GTMP kinases are different from classical kinases in that such a lysine is absent in the motif II p-loop, ${ }^{10}$ except for PMK, which contains a lysine at the end of the p-loop. However, we have previously found that a positively charged residue (Arg228) in galactokinase can reorientate toward ATP to facilitate the phosphoryl transfer. ${ }^{10}$ Similar to Arg228 in galactokinase, Lys101 functions to stabilize the negative charge developed at the $\beta$-, $\gamma$-bridging oxygen atom of ATP during phosphoryl transfer.

The RMSD graph of the MD simulation of K101R fluctuated in the first 5 ns and became stabilized at approximately $1.2 \AA$, indicating that the system remained stable and had reached equilibrium after 5 ns. When lysine was altered to arginine in K101R, one of the coordinated water molecules moved far away from $\mathrm{Mg}^{2+}$ disrupting the coordination of the ion. This observation is in consistent with the functional role of conserved lysine of classical kinases in orienting and facilitating the phosphate transfer. ${ }^{40}$ 


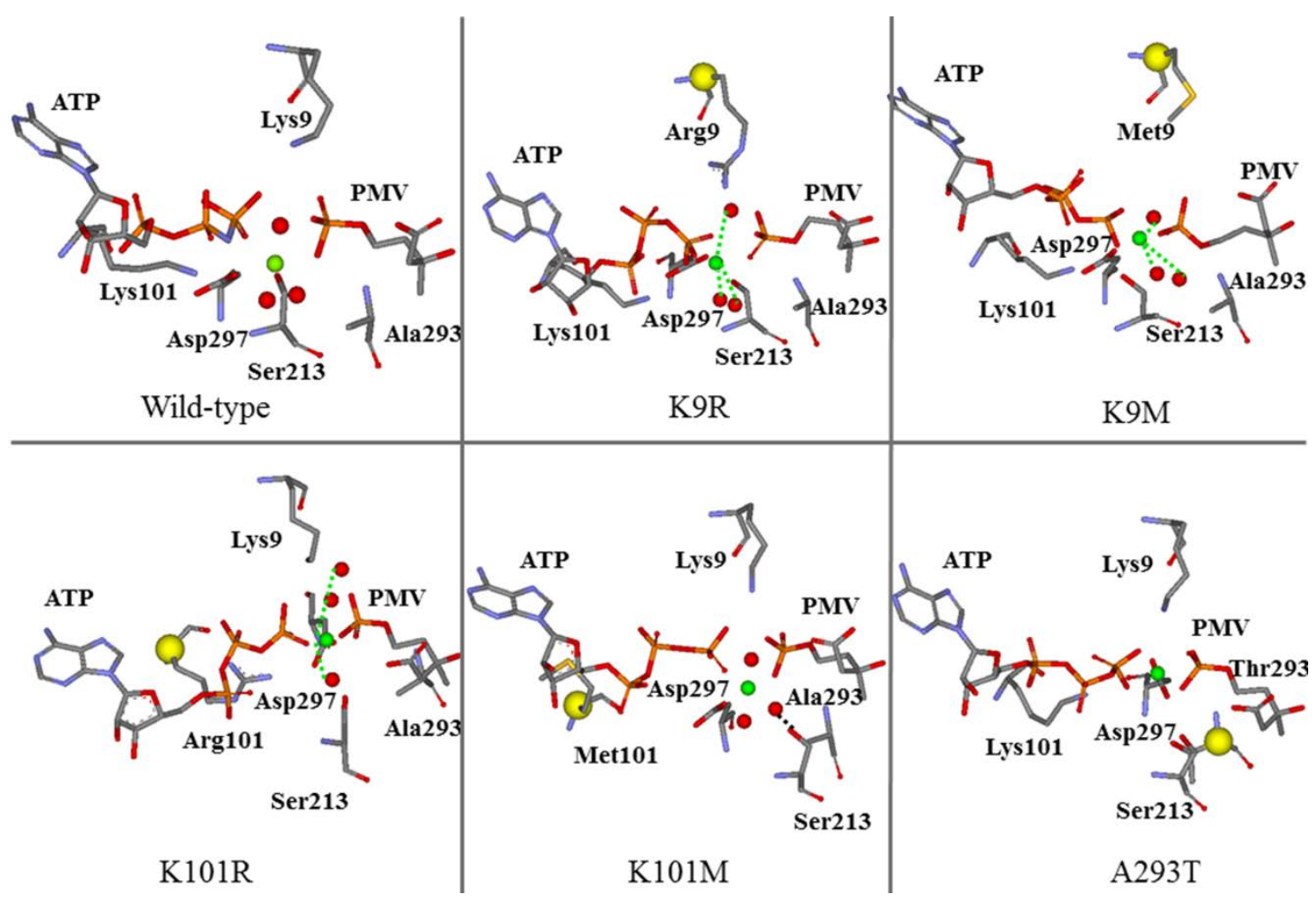

Figure 5. Close up view of the catalytic sites of WT PMK and the mutated kinase. Structures were obtained by 10-15ns MD simulations.

The protein backbone in the MD simulation of K101M fluctuated majorly in the first $10 \mathrm{~ns}$, which indicates that significant conformational changes occurred in the system with this alteration. The RMSD value slightly decreased after 10 ns and finally stabilized around 1.2 $\AA$ from 12 ns. Ser213 is pushed away from active site when Lys101 was replaced to methionine. This suggests Lys101 helps to grasp Ser213 around the substrate phosphoryl group to facilitate the proton transfer during phosphorylation.

In the work of Andreassi, J. L et al. alteration of Ala293 on the steady-state kinetic

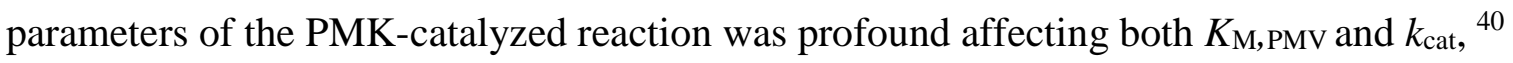
indicating this residue has significant influence on the binding affinity of the enzyme for the substrate as well as the catalytic efficiency of the enzyme. In the MD simulation of A293T, the protein backbone remained fairly stable during the 8 ns simulation fluctuating slightly by approximately $1.0 \AA$. The threonine at position 293 formed a H-bond with the catalytic residue Asp297, replacing the original H-bond network between Asp297 and three coordinating water molecules in the crystal structure (Figure S4), as a result, all the three coordinating water molecules moved away from the active site and the coordination of $\mathrm{Mg}^{2+}$ 
was completely disrupted. This indicates Ala293 plays a significant role in the catalytic reaction by maintaining the space requested the coordinating water and therefore the octahedral coordination of the magnesium ion.

\subsection{Reaction pathway of PMK by QM/MM study}

In the MD simulation of WT PMK, we observed that Ser213 on the $\alpha$-helix at the catalytic site is repositioned to further approach the substrate. We propose the resulting tight H-bond network would facilitate the proton transfer from the hydroxyl group of the substrate to the $\gamma$ phosphoryl group of ATP. Therefore, this residue was included in the QM region, along with other previously identified catalytically important residues Ala293, Lys9 and Lys101. The substrate PMV, Asp297, and three coordinated water molecules that are coordinated to the $\mathrm{Mg}$ ion were also included in the QM region (Figure 1). In the QM/MM calculations on the phosphorylation reaction of protein kinase A (PKA), the triphosphate arm of ATP and the entire ATP molecule were included in the QM region. ${ }^{46}$ Both of the results were approximately consistent with the experimental estimation, indicating that the triphosphate arm of ATP in QM should be sufficient and efficient in the study of catalytic mechanism. Thus it is not always necessary to have a large QM region to minimize the difference between the theoretical and the experimental energies due to the additional approximations for a large QM/MM boundary. Therefore, here we only kept the triphosphate arm of ATP in the QM region.

Electronic embedding incorporates the partial charges of the MM region into the quantum mechanical Hamiltonian. This technique provides a better description of the electrostatic interaction between the QM and MM regions than the mechanical embedding method and allows the QM wave function to be polarized by the MM environment. ${ }^{47}$

Ten representative structures were selected from the wild-type MD simulation. All of these structures were optimized by QM/MM to decide the suitable starting structure for the subsequent potential energy scan (Figure S5). Important interatomic distances of these structures are listed in Table S2. Cluster7 was selected for a further potential energy surface scan. The adiabatic mapping approach was used to delineate the reaction path and locate stationary points such as transition state and product. ${ }^{48}$ A two-dimensional potential energy surface (PES) scan was calculated to understand the conformational change along reaction path by defining the distances of $\mathrm{d}_{1}=\mathrm{d}\left(\mathrm{P}^{\gamma}-\mathrm{O}^{\beta 3}\right)$ and $\mathrm{d}_{2}=\mathrm{d}\left(\mathrm{P}^{\gamma}-\mathrm{O}^{\mathrm{P} 3}\right)$ as the reaction coordinates. 
The $k_{\text {cat }}$ value is $3.4 \mathrm{~s}^{-1}$ at $310 \mathrm{~K}$ for PMK from $S$. pneumoniae, ${ }^{49}$ corresponding to an activation energy of $17.34 \mathrm{kcal} / \mathrm{mol}$. By the QM/MM method a transition state was located with $\mathrm{d}_{1}=2.27 \AA$ and $\mathrm{d}_{2}=2.08 \AA$ and was validated by a unique imaginary frequency of -140.62 $\mathrm{cm}^{-1}$ that corresponds to the $\gamma$-phosphate transfer to PMV (Figure 6). The transition state was optimized employing mechanical embedding and associated with a potential energy barrier of $15.87 \mathrm{kcal} / \mathrm{mol}$ (Figure 7), which is in good agreement with the kinetic data.

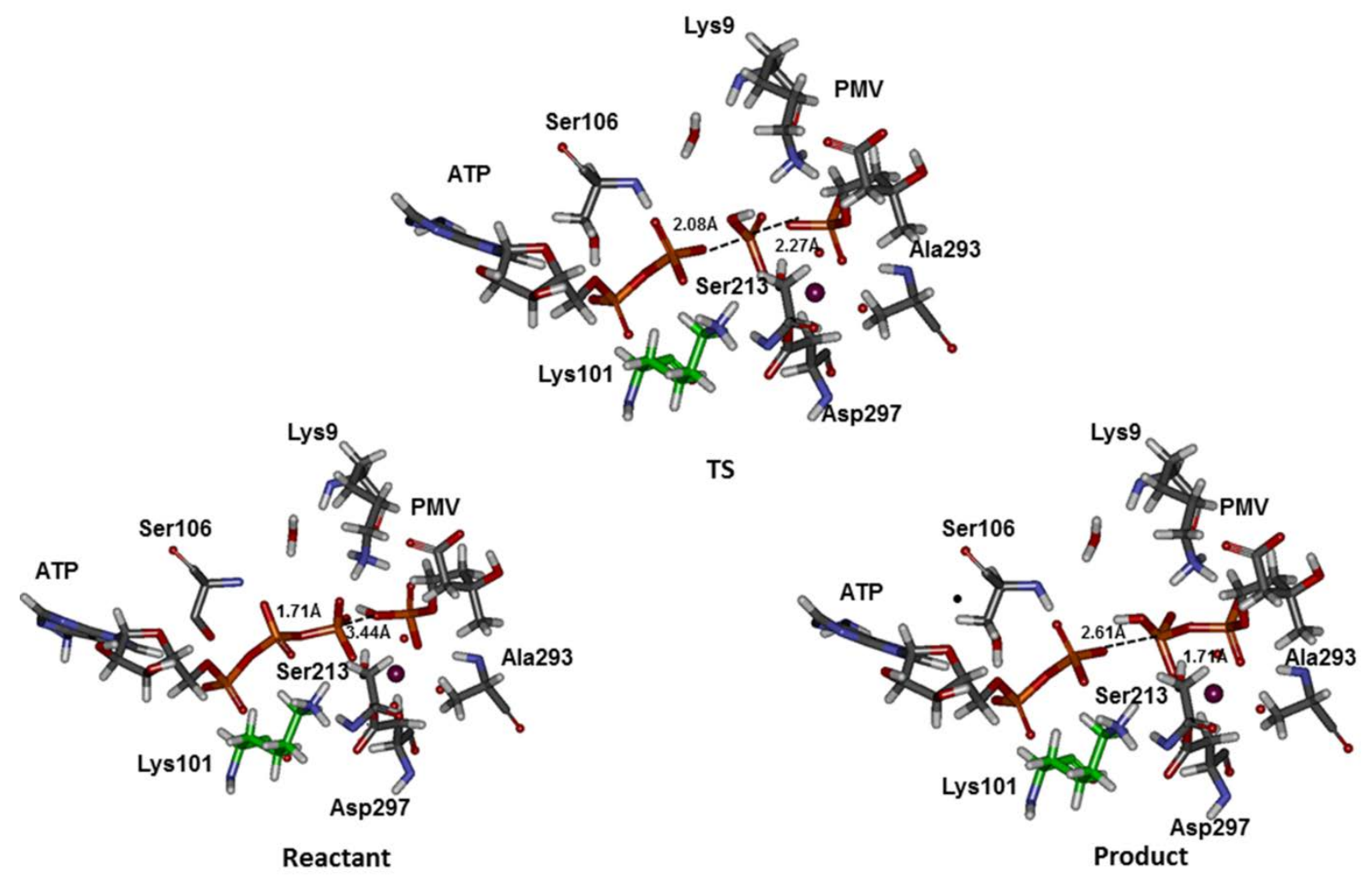

Figure 6. The QM/MM optimized structures of the reactant, transition state (where $\mathrm{d}_{1}=2.27$ $\AA, \mathrm{d}_{2}=2.08 \AA$ ) and product. 

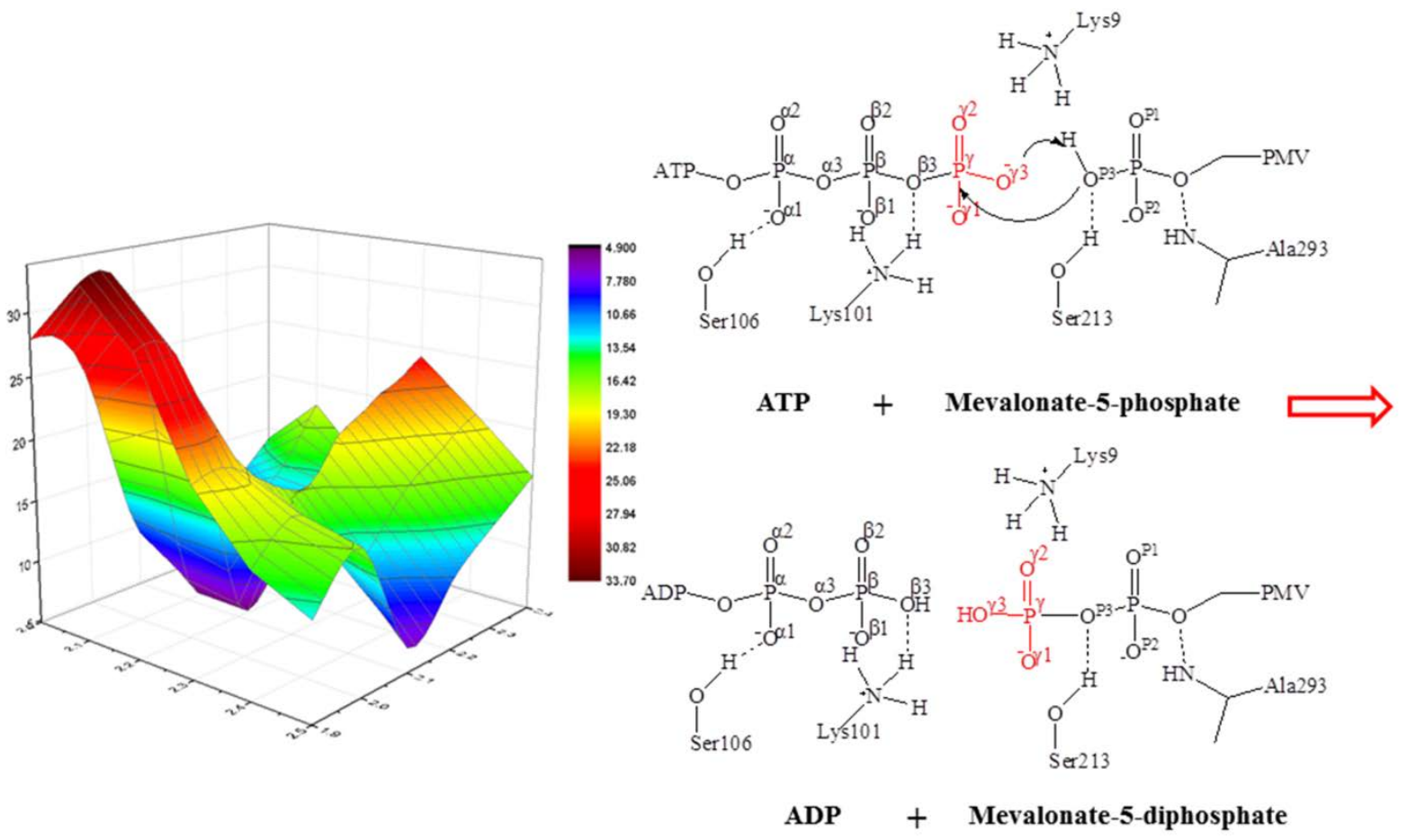

Figure 7. The potential energy surface scan, with the distance $d_{1}=d\left(P^{\gamma}-O^{\beta 3}\right)$ and $d_{2}=d\left(P^{\gamma}-O^{P 3}\right)$ as the reaction coordinates, yields an activation barrier of $15.87 \mathrm{kcal} / \mathrm{mol}$ Pauling's formula ${ }^{50}: \mathrm{D}(\mathrm{n})=\mathrm{D}(\mathrm{l})-0.6 \log n$ was used to estimate associative or dissociative character of the transition state. Here, $\mathrm{D}(\mathrm{l})$ is $1.73 \AA$ for the $\mathrm{P}-\mathrm{O}$ bond and $\mathrm{D}(\mathrm{n})$ is the average of $\mathrm{rO}_{\mathrm{O} 1-\mathrm{P} \gamma}$ and $\mathrm{rP}_{\mathrm{P}-\mathrm{O} \beta}$ at the transition state. The fractional bond number (n) is 0.18 , which gives a dissociative character of $82 \%$. According to the transition state structure disclosed by QM/MM calculations, a possible mechanism for PMK was proposed (Figure 8). While the substrate gradually approaches ATP, the hydroxyl hydrogen atom of the phosphate in the substrate $\left(\mathrm{H}-\mathrm{O}^{\mathrm{P} 3}\right)$ migrates to $\gamma$-phosphate oxygen atom $\left(\mathrm{O}_{\gamma 3}\right)$ of ATP. The backbone amino group of Ala293 is hydrogen-bonded with phosphoryl oxygen $\left(\mathrm{O}^{\mathrm{P} 2}\right)$ atom of PMV, facilitating the proton transfer from the substrate to the $\gamma$-phosphate of ATP. Lys9 is proposed to stabilize both the transition state and product conformation. Lys101 is suggested to help neutralizing the negative charge developed at the $\beta$-, $\gamma$ - bridging oxygen of ATP during phosphorylation. 


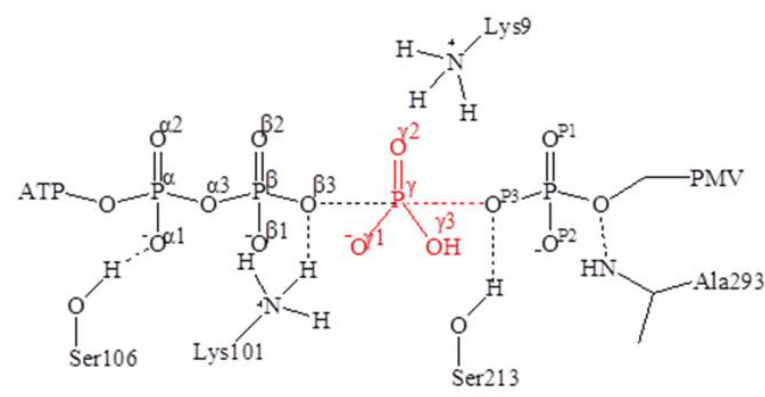

TS

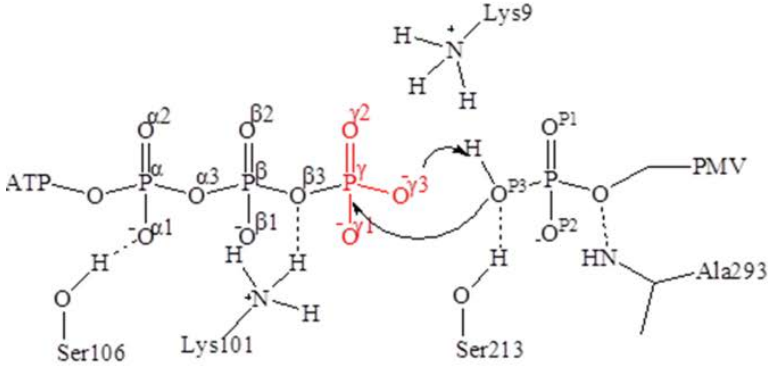

$\mathbf{R}$

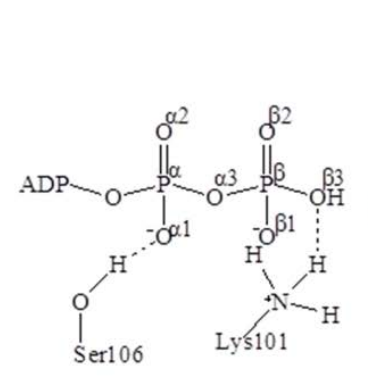

(1)

$\mathbf{P}$

Figure 8. Proposed phosphorylation mechanism of PMK.

\section{Conclusions}

PMK, a member of GHMP family was studied in detail using MD simulations and hybrid QM/MM calculations. Important catalytic residues were identified, including a highly conserved residue (Lys9) that stabilizes the phosphoryl groups of the substrate and ATP and Ala293 that tethers the substrate by a hydrogen bond with the phosphoryl group. These observations are all in agreement with enzymological and mutagenesis studies on this kinase. We found that the highly conserved Lys101 near the ATP binding site stabilizes the negative charge that develops at the $\beta$-, $\gamma$-bridging oxygen atom of ATP during the cleavage of the P-O bond. In addition, a conserved serine residue on the p-loop, Ser106, reorientates to anchor the nucleotide in the catalytic site. Moreover, Ser213 on the $\alpha$-helix at the catalytic site further approaches the substrate to form a tight H-bond network with the hydroxyl oxygen of the protonated phosphoryl group, facilitating the proton transfer during phosphorylation.

Based on the protein structure and current results obtained in this research, the mechanism of PMK is believed to follow a direct attack pathway and the phosphoryl transfer occurs through a dissociative-dominated mechanism. A potential transition state structure was located, which was validated by a unique imaginary frequency. Thus despite of the different configuration of the active sites of GHMP kinases and divergent substrates, it appears (that) many, perhaps all, 
GHMP kinases follow a direct phosphorylation mechanism. This research discloses the reaction mechanism of PMK, a representative kinase in the MVA pathway. It has potential impact in both drug discovery and biotechnology. In particular these studies have identified catalytically important residues which could be targeted by MVK inhibitors in the search for novel antibiotics. They may also provide guidance for the engineering attempts to alter or broaden the substrate specificity of MVK.

\section{Acknowledgements}

The authors acknowledge the financial support from INVEST NI RD0314092. We also would like to thank the information service at Queen's University Belfast for the high performance computing resources.

\section{Supporting Information}

The Supporting Information is available free of charge on the ACS Publications website. Figures S1-S5; Tables S1 and S2; coordinates of the transition state (PDB)

\section{References}

(1) Bork, P.; Sander, C.; Valencia, A. Convergent Evolution of Similar Enzymatic Function on Different Protein Folds: the Hexokinase, Ribokinase, and Galactokinase Families of Sugar Kinases. Protein Sci. 1993, 2, 31-40.

(2) Potter, D.; Wojnar, J.M.; Narasimhan, C.; and Miziorko, H.M. Identification and Functional Characterization of an Active-Site Lysine in Mevalonate Kinase. J. Biol. Chem. 1997, 272, 5741-5746.

(3) Potter, D.; and Miziorko, H. M. Identification of Catalytic Residues in Human Mevalonate Kinase. J. Biol. Chem. 1997, 272, 25449-25454.

(4) Reinhardt, L.A.; Thoden, J.B.; Peters, G.S.; Holden, H.M.; Cleland, W.W. pH-Rate Profiles Support a General Base Mechanism for Galactokinase (Lactococcus lactis). FEBS Lett. 2013, 587, 2876-81.

(5) Chu, X.; Li, N.; Liu, X.; Li, D. Functional Studies of Rat Galactokinase. J. Biotechnol. 2009, 141, 142-146. 
(6) Megarity, C.F.; Huang, M.; Warnock, C. and Timson, D.J. The Role of the Active Site Residues in Human Galactokinase: Implications for the Mechanisms of GHMP Kinases. Bioorganic chemistry 2011, 39, 120-126.

(7) Miziorko, H.M. Identification of Active Site Residues in Mevalonate Diphosphate Decarboxylase: Implications for Family of Phosphotransferases. Protein Science 2004, $13,1875-1881$.

(8) Zhou, T.; Daugherty, M.; Grishin, N. V.; Osterman, A L. \& Zhang, H. Structure and Mechanism of Homoserine Kinase: Prototype for the GHMP Kinase Superfamily. Structure 2000, 8, 1247-1257.

(9) Krishna, S. S.; Zhou, T.; Daugherty, M.; Osterman, A. \& Zhang, H. Structural Basis for the Catalysis and Substrate Specificity of Homoserine Kinase. Biochemistry 2001, 40, 10810-10818.

(10) Huang, M.; Li, X.; Zou, J. W. and Timson D. J. Role of Arg228 in the Phosphorylation of Galactokinase: The Mechanism of GHMP Kinases by Quantum Mechanics/Molecular Mechanics Studies. Biochemistry 2013, 52, 4858-4868.

(11) Timson, D. J. GHMP Kinases-Structures, Mechanisms and Potential for Therapeutically Relevant Inhibition. Current Enzyme Inhibition 2007, 3, 77-94. Chou, H.H.; Keasling, J.D. Synthetic Pathway for Production of Five-Carbon Alcohols from Isopentenyl Diphosphate. Appl. Environ. Microbiol. 2012, 78, 78497855.

Wilding, E.I.; Brown, J.R.; Bryant, A.P.; Chalker, A.F.; Holmes, D.J.; Ingraham K.A.; Iordanescu S.; So C.Y.; Rosenberg M.; Gwynn M.N. Identification, Evolution, and Essentiality of the Mevalonate Pathway for Isopentenyl Diphosphate Biosynthesis in Gram-Positive Cocci. J Bacteriol. 2000, 182, 4319-27.

(14) Kudoh, T.; Park, C.S.; Lefurgy, S.T.; Sun, M.; Michels, T.; Leyh, T.S.; Silverman, R.B. Mevalonate Analogues as Substrates of Enzymes in the Isoprenoid Biosynthetic Pathway of Streptococcus Pneumoniae. Bioorg Med Chem. 2010, 18, 1124-34. Lefurgy, S.T.; Rodriguez, S.B.; Park, C.S.; Cahill, S.; Silverman, R.B.; Leyh, T.S. Probing Ligand-Binding Pockets of the Mevalonate Pathway Enzymes from Streptococcus Pneumoniae. J Biol Chem. 2010, 285, 20654-63.

$$
\text { Silverman, R.B.; Kudoh, T. Inhibitors of the Mevalonate Pathway of }
$$

Streptococcus Pneumonia. 2014, US 8710032 B2. 
Natural Product Drug Discovery and Development. Med. Res. Rev. 1998, 18, 315-331.

$$
\text { Dhingra, V.; Rao, K.V. \& Narasu, M.L. Current Status of Artemisinin and its }
$$

Derivatives as Antimalarial Drugs. Life Sci. 2000, 66, 279-300.

$$
\text { George, K.W.; Alonso-Gutierrez, J.; Keasling, J.D.; Lee, T.S. Isoprenoid }
$$

Drugs, Biofuels, and Chemicals-Artemisinin, Farnesene, and Beyond. Advances in Biochemical Engineering/Biotechnology 2015, 148, 355-389.

Gupta, P.; Phulara, S.C. Metabolic Engineering for Isoprenoid-Based Biofuel Production. J Appl Mocrobilogy 2015, 119, 605-619.

(21) Chang, M.C.Y. and Keasling, J.D. Production of Isoprenoid Pharmaceuticals by Engineered Microbes. Nat. Chem. Biol. 2006, 2, 674-681.

(22) George, K.W.; Thompson, M.G.; Kang, A.; Baidoo, E.; Wang, G.; Chan, L.J.G.; Petzold, C.J.; Adams, P.D.; Keasling, J.D.; Lee, T.S. Metabolic Engineering for the High Yield Production of Isoprenoid-Based C5 Alcohols in E. Coli. Sci. Rep. 2015, 5:11128. DOI: 10.1038/srep11128.

Zhu, F.; Zhong, X.; Hu, M.; Lu, L.; Deng, Z.; Liu, T. In Vitro Reconstitution of Mevalonate Pathway and Targeted Engineering of Farnesene Overproduction in Escherichia Coli. Biotechnol. Bioeng. 2014, 111, 1396-1405.

(24) Zheng, Y.; Liu, Q.; Li, L.; Qin, W.; Yang, J.; Zhang, H.; Jiang, X.; Cheng, T.; Liu, W.; Xu, X. et al. Metabolic Engineering of Escherichia Coli for Highspecificity Production of Isoprenol and Prenol as Next Generation of Biofuels. Biotechnol Biofuels. 2013, 6: 57.

(25) Redding-Johanson, A.M.; Batth, T.S.; Chan, R.; Krupa, R.; Szmidt, H.L.; Adams, P.D.; Keasling, J.D.; Lee, T.S.; Mukhopadhyay, A.; Petzold, C.J. Targeted Proteomics for Metabolic Pathway Optimization: Application to Terpene Production. Metab. Eng. 2011, 13, 194-203. Anthony, J.R.; Anthony, L.C.; Nowroozi, F.; Kwon, G.; Newman, J.D.; Keasling, J.D. Optimization of the Mevalonate-Based Isoprenoid Biosynthetic Pathway in Escherichia Coli for Production of the Anti-Malarial Drug Precursor Amorpha-4,11-Diene. Metab. Eng. 2009, 11, 13-19.

(27) Martin, V.J.; Pitera, D.J.; Withers, S.T.; Newman, J.D.; Keasling, J.D. Engineering a Mevalonate Pathway in Escherichia Coli for Production of Terpenoids. Nat Biotechnol. 2003, 21, 796-802. 
Andreass, J. L. II; Vetting, M. W.; Bilder, P. W.; Roderick, S. L. \& Leyh, T.S. Structure of the Ternary Complex of Phosphomevalonate Kinase: the Enzyme and its Family. Biochemistry 2009, 48, 6461-6468.

(29) Frisch, M. J.; Trucks, G. W.; Schlegel, H. B.; Scuseria, G. E.; Robb, M. A.; Cheeseman, J. R.; Scalmani, G.; Barone, V.; Mennucci, B.; Petersson, G. A. et al. 2009, Gaussian 09. Bayly, C. I.; Cieplak, P.; Cornell, W. and Kollman, P. A. A Well-Behaved Electrostatic Potential Based Method Using Charge Restraints for Deriving Atomic Charges: the RESP model. J. Chem. Phys. 1993, 97, 10269-10280.

Case, D.A.; Darden, T.A.; Cheatham, T.E. III; Simmerling, C.L.; Wang, J.; Duke, R.E.; Luo, R.; Crowley, M.; Walker, R.C.; Zhang, W. et al. 2008, AMBER 10, University of California, San Francisco.

Wang, J.; Cieplak, P. and Kollman, P. A. How Well Does a Restrained Electrostatic Potential (RESP) Model Perform in Calculating Conformational Energies of Organic and Biological Molecules? J. Comput. Chem. 2000, 21, 10491074. Darden, T.; York, D. and Pedersen, L. Particle Mesh Ewald: An N·log(N) Method for Ewald Sums in Large Systems. J. Chem. Phys. 1993, 98, 10089-10092. Ryckaert, J. P.; Ciccotti, G. \& Berendsen, H. J. C. Numerical Integration of the Cartesian Equations of Motion of a System with Constraints: Molecular Dynamics of n-Alkanes. J. Comput. Chem. 1977, 23, 327-341.

Shao, J.; Tanner , S.W.; Thompson, N.; Cheatham, T.E. Clustering Molesular Dynamics Trajectories:1. Characterizing the Performance of Different Clustering Algorithms. Journal of Chemical Theory and Computation 2007, 3, 2312-2334.

(36) Vreven, T.; Byun, K. S.; Komáromi, I.; Dapprich, S.; Montgomery Jr., J.

A.; Morokuma, K. and Frisch, M. J. Combining Quantum Mechanics Methods with Molecular Mechanics Methods in ONIOM. J. Chem. Theory Comput. 2006, 2, 815826.

(37) Singh, U. C. \& Kollman, P. A. An Approach to Computing Electrostatic Charges for Molecules. J. Comput. Chem. 1984, 5, 129-145.

Besler, B. H. Jr.; K. M. M. \& Kollman, P. A. Atomic Charges Derived from Semiempirical Methods. J. Comput. Chem. 1990, 11, 431-439. 
Matrix Metalloproteinase 2 Inhibition: Combined Quantum Mechanics and Molecular Mechanics Studies of the Inhibition Mechanism of (4-Phenoxyphenylsulfonyl) methylthiirane and its oxirane analogue. Biochemistry 2009, 48, 9839-9847. Andreassi, J. L. \& Leyh, T. S. Molecular Functions of Conserved Aspects of the GHMP Kinase Family. Biochemistry 2004, 43, 14594-14601.

(41) Shen, Q.; Wang, G.; Li, S.; Liu, X.; Lu, S.; Chen, Z.; Song, K.; Yan, J.; Geng, L.; Huang, Z. et al. ASD v3.0: Unraveling Allosteric Regulation with Structural Mechanisms and Biological Networks. Nucleic Acids Res. 2016; 44(D1): D527-35.

(42) Shi, F.; Telesco, S. E.; Liu, Y.; Radhakrishnan, R. \& Lemmon, M. A ErbB3/HER3 Intracellular Domain is Competent to Bind ATP and Catalyze Autophosphorylation. Proc. Natl. Acad. Sci. USA. 2010, 107, 7692-7697.

Shi, T.; Lu, Y.; Liu, X.; Chen, Y.; Jiang, H. and Zhang, J. Mechanism for the Autophosphorylation of CheAhistidine Kinase: QM/MM Calculations. J. Phys. Chem. B. 2011, 115, 11895-11901.

Transfer Mechanism of Cyclin-Dependent Protein Kinases from Ab Initio QM/MM Free-Energy Studies. J. Phys. Chem. B. 2011, 115, 13713-13722.

(45) Carrera A.C.; Alexandrov K.; Roberts T.M. The Conserved Lysine of the Catalytic Domain of Protein Kinases is Actively Involved in the Phosphotransfer Reaction and not Required for Anchoring ATP. Proc. Natl. Acad. Sci. USA. 1993, 90, 442-446.

(46) Cheng, Y.; Zhang, Y. and McCammon, J. A. How Does the cAMP-

Dependent Protein Kinase Catalyze the Phosphorylation Reaction: An ab Initio QM/MM Study. J. Am. Chem. Soc. 2005, 127, 1553-1562.

$$
\text { Chung, L.W.; Sameera, W.M.; Ramozzi, R.; Page, A.J.; Hatanaka, }
$$

M.; Petrova, G.P.; Harris, T.V.; Li, X.; Ke, Z.; Liu, F. et al. The ONIOM Method and Its Applications. Chem Rev. 2015, 115, 5678-796.

(48) Lonsdale, R.; Harvey, J. N. \& Mulholland, A. J. A practical Guide to Modelling Enzyme-Catalysed Reactions. Chem. Soc. Rev. 2012, 41, 3025-3038. Pilloff, D.; Dabovic, K.; Romanowski, M.J.; Bonanno, J.B.; Doherty, M.; Burley, S.K.; Leyh, T.S. The Kinetic Mechanism of Phosphomevalonate Kinase. J. Biol. Chem. 2003, 278, 4510-4515. 
(50) Mildvan, A.S. Mechanisms of Signalling and Related Enzymes.

Proteins. 1997, 29, 401-16.

TOC Graphic 


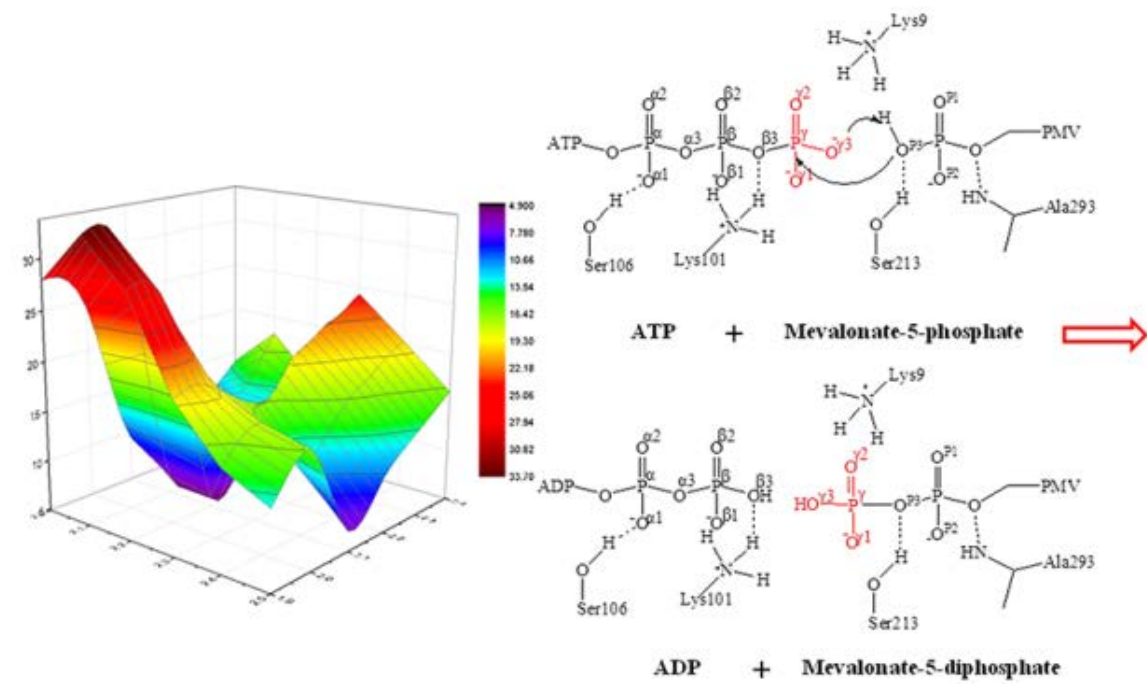

